\title{
La relación entre la escuela, la salud y la legislación para el logro de una educación antitabáquica
}

\section{The relationship between school, health and legislation for the achievement of an anti-tobacco education}

\author{
Karel Llopiz Guerra \\ Universidad Central "Marta Abreu" de las Villas, Cuba \\ ORCID: https://orcid.org/0000-0002-1500-8000 \\ Francis Díaz Flores \\ Universidad Nacional Mayor de San Marcos, Perú \\ ORCID: https://orcid.org/0000-0002-9272-9995 \\ Liset Sulay Rodriguez Baca \\ Universidad Autónoma del Perú, Perú \\ ORCID: https://orcid.org/0000-0003-1850-615X \\ Nadia Aguilar - Hernando \\ Universidad Peruana Los Andes, Perú \\ ORCID: https://orcid.org/0000-0001-9883-4596
}

*Correspondence

Email: kllopiz@uclv.cu
Cite as:

Llopiz, K., Díaz, F., Rodriguez, L., \& Aguilar-Hernando, N. (2021). La relación entre la escuela, la salud y la legislación para el logro de una educación antitabáquica. Propósitos y Representaciones, $9(1), \quad$ e1058. Doi: http://dx.doi.org/10.20511/pyr2021.v9n1.1058 


\title{
Resumen
}

Ante circunstancias adversas, numerosas personas han buscado refugio en las adicciones a las drogas, el uso y abuso de las mismas, lo que trae consigo consecuencias negativas para su salud. Las autoridades y profesionales de diferentes países han desarrollado un grupo de acciones en los órdenes educativo, jurídico, social y ambiental, y han declarado una lucha muy marcada para la prevención ante las drogas legales. El presente artículo tiene como objetivo: Caracterizar el vínculo intersectorial sobre el tabaquismo y su educación, atenuación y control a partir de un estudio ante la situación en el mundo contemporáneo.

Palabras claves: escuela, salud, jurídico, educación antitabaquica.

\begin{abstract}
Faced with adverse circumstances, many people have sought refuge in drug addictions, drug use and abuse, which have negative consequences for their health. The authorities and professionals of different countries have developed a group of actions in the educational, legal, social and environmental fields, and have declared a very marked fight for the prevention of legal drugs. The aim of this article is to characterize the intersectoral link on smoking and its education, attenuation and control based on a study of the situation in the contemporary world.
\end{abstract}

Keywords. School, healt, legal, antitabaquic education.

\section{Introducción}

En el mundo actual, las condiciones de vida se han hecho más complejas, a partir de enfrentar una crisis general que repercute en los problemas sociales, dentro de los cuales se encuentran la acentuación de los riesgos de salud.

El tabaquismo o hábito de fumar es una de las causas prevenibles de defunción en el planeta y uno de los principales problemas de salud pública, es la epidemia que más causa mortalidad en el mundo y hoy se reconoce como la pandemia del siglo XXI; es una enfermedad crónica adictiva potencialmente curable y que mata al $50 \%$ de los consumidores. Martín, et-al (2015).

El tabaquismo cobra vidas y según estudios realizados cada seis segundos esta fatal consecuencia ocurre, siendo una causa de muerte prevenible. Seis millones de personas mueren cada año en el planeta a causa de esta enfermedad- adición no transmisible. En las asambleas internacionales de salud, se concibe la proyección de estrategias para mejorar los estilos de vida, poder prevenir enfermedades evitables y rehabilitar a las personas.

El tabaco es una droga legal con efectos simultáneos estimulantes y depresores, cuyo consumo frecuente conduce a la tolerancia y dependencia con la determinación de consecuencias nocivas sobre el sujeto, la sociedad o ambos. Calleja (2010) 
La problemática del consumo de tabaco en el orbe, constituye un aspecto de vital atención para todos los países. Esta acción dirigida por la Organización Mundial de la Salud (OMS). Fumar se encuentra arraigado desde la antigüedad en las costumbres y cultura de poblaciones y aun existiendo leyes que regulen sus formas de consumo y espacios para desarrollarlos, en no pocas ocasiones se violan estas normativas por parte de los consumidores.

Se debe tener en cuenta que este problema del orbe, necesita de decisiones y acciones uniformes para dar solución a esta situación y desde un enfoque social, también debe lograrse una perspectiva de percepción de riesgo, necesitando que se genere un cambio social ante este flagelo. Amplio impacto ha tenido la puesta en vigor tanto del CMCT OMS, como del plan de medidas MPOWER. OMS (2015).

Las acciones que se deben realizar para la prevención del tabaquismo deben involucrar a maestros, padres, organizaciones sociales, profesionales de la salud, e incluso, estudiantes de ciencias médicas. En el accionar de estos últimos las actividades extensionistas juegan un papel protagónico. Llopiz (2018)

El presente artículo tiene como objetivo: Caracterizar el vínculo intersectorial sobre el tabaquismo y su educación, atenuación y control a partir de un estudio ante la situación actual en el mundo.

\section{Argumentación}

Los resultados de las investigaciones en el campo del enfrentamiento al tabaquismo constituyen una importante fuente de información para conocer comportamientos, efectos y resultados de las acciones que se emprenden por los diferentes países para desarrollar intervenciones efectivas. Pupo, Hechevarria, Lozano. (2016)

La Organización Mundial de la salud (OMS), ha pedido a la comunidad mundial que se aumenten tributos de $75 \%$ a los precios del cigarrillo, al considerarse que a pesar de existir aproximadamente 1.000 millones de personas fumadores en el mundo, solo 33 países han aumentado sus medidas, resaltando dentro de estos China y Francia, los cuales aumentaron los impuestos, dándoles como resultando lograr una disminución del tabaquismo y de las enfermedades relacionadas, como es el caso del cáncer de pulmón. A estos les sigue Filipinas, que en los últimos años ha incrementado sus impuestos a más de $300 \%$. OMS (2015).

Desde hace años, los países que conforman la Organización Mundial de la Salud, como parte de sus políticas públicas abogan por el logro del "Marco para la Lucha Antitabáquica".

Desde la Atención Primaria, se debe continuar profundizando en acciones vinculadas al tratamiento en las personas interesadas en lograr la deshabituación del hábito, y continuar la vigilancia epidemiológica que permita desde las investigaciones y estudios la atenuación y control del tabaquismo. 
El carácter eminentemente social de la educación y su relación con la preparación cultural de las personas y la necesaria consideración del entorno para la preparación profesional de los docentes para atender la salud como punto de partida y resultado de una educación de calidad, obliga a orientar el trabajo en esa dirección con vistas a resolver problemas concretos vinculados con la práctica social en general y profesional. Llopiz (2018)

Resulta interesante la experiencia de acciones prohibitivas en países europeos como: Irlanda, Noruega, Italia y España, donde está sancionado el consumo de tabaco en bares y restaurantes. Otro país con experiencias positivas para establecer un mayor control y promoción en contra del tabaquismo, ha sido Australia, donde las tabacaleras multinacionales vieron en peligro sus ganancias a partir de que la severa ley anti tabáquica, que obligaba a presentar los cigarrillos en paquetes homogéneos y sin publicidad y las advertencias sobre los riesgos de fumar para la salud, ocuparían el $75 \%$ de la parte frontal de los envoltorios Baskerville, B. et. al. (2015).

En América el tabaco en particular tiene gran aceptación social y ha alcanzado proporciones muy grandes, no solo por el número de fumadores en el planeta, sino también por las graves consecuencias que trae contra la salud y los costos sociales. León, Ferreira, Pillon (2010)

La oferta accesible de productos del tabaco, ha sido una de las causas que, presenta un incremento sostenido del consumo de cigarrillos. Suárez (2016). Cuba se encuentra entre los países con una alta prevalencia de consumidores.

A partir de las acciones de prevención y promoción de salud en Cuba desde 1985 se desarrollaron campañas para la deshabituación tabáquica, a partir de la tendencia creciente de la prevalencia y el consumo. Y desde hace varias décadas, en las propias cajas de cigarros existe información sobre la nocividad del hábito por los medios de comunicación y varios especialistas del sector, donde se ha logrado, desde 1998 iniciar la labor de la Comisión Nacional de Promoción de Salud.

Aun con esta premisa, desde la normativa jurídica, Cuba para la reducción de la demanda, posee solo seis instrumentos jurídicos con medidas económicas y las mismas no persiguen la prevención y control del tabaquismo. Las medidas dirigidas a limitar la accesibilidad al cigarrillo, fundamentalmente en los adolescentes y jóvenes, así como reducir los lugares donde resulta factible fumar, permiten otorgar coherencia y acompañamiento a la educación que se realiza. Lograr la no aceptación social del tabaquismo se convierte en una premisa para su prevención y control. Suárez, Galcerán (2018). En Cuba, la prevalencia del tabaquismo ha sido considerada alta desde que se dispone de información al respecto. Suarez (2015).

En los centros educacionales cubanos se realizan actividades encaminadas a prevenir el consumo de sustancias adictivas, las cuales deben ser sistematizadas y combinadas con la autopreparación de los estudiantes. Estas responden a orientaciones del Ministerio de Educación, de Educación Superior, así como de Salud Pública. Rodríguez, Cruz (2015). 
A nivel mundial la estrategia para el logro de una educación antitabáquica en las poblaciones se debe expresar desde una concepción multisectorial y multidisciplinaria, basada en acciones educativas dirigidas a la niñez y la juventud, por ser la población más vulnerable ante esta enfermedad adicción, donde las diferentes acciones desde la legalidad y en el orden educativo permita las orientaciones dirigidas a la población en general y sobre todo la que se encuentra expuesta como grupos de riesgos.

Por lo que, mantener la prohibición de la publicidad directa e indirecta de los productos del tabaco, las advertencias sanitarias, la prohibición de fumar en lugares públicos y de servicios, así como la prohibición de la venta a menores de edad, son de las acciones que permiten lograr la lucha contra el tabaquismo.

\section{Conclusiones}

La investigación realizada permite concluir, que desde la educación, las instituciones de salud deben hacer cumplir con los aparatos y las normativas jurídicas vigentes para la prevención y el control del tabaquismo a nivel mundial, aun cuando existen con fuerza un cuerpo legal en regiones y países, determinadas políticas gubernamentales, estrategias en el ámbito de la salud y en los sistemas educativos, distan en hacerlas cumplir a partir de las aspiraciones deseadas.

Las medidas económicas propuestas necesitan aplicarse con mayor fuerza y debe hacerse cumplir con el objetivo de atenuar y erradicar las diferentes formas de obtención de cigarros, principalmente en los adolescentes, jóvenes y en un no desestimado número de niños, factores que alertan la necesidad de continuar la lucha antitabaquica.

\section{Referencias}

Calleja, N. (2010). Susceptibilidad al consumo del tabaco y comportamiento tabáquico en las adolescentes. Univ Psicol. Vol 11, (4). Disponible en: http://www.scielo.org.co/scielo.php?script=sci_arttext\&pid=S1657 92672012000400016\&lng=en\&nrm=iso

Cruz, A. Llopiz, K. Molerio, P. (2019). Educación antitabáquica, percepción de riesgo e incidencia en el rendimiento académico de estudiantes universitarios de Ciencias Médicas. Vol. $\quad 8, \quad \mathrm{~N}^{\circ} \quad 1 . \quad$ Disponible en: https://revistas.usil.edu.pe/index.php/pyr/article/view.

Martín, S. M. et-al (2015). Comportamiento del tabaquismo en los adolescentes del Reparto Fructuoso Rodríguez, Cárdenas. Rev. Med Electrón. Disponible en: http://scielo.sld.cu/scielo.php?script=sci_arttext\&pid=S168418242015000600004

Organización Mundial de la Salud. (2015). MPOWER y otros recursos. Geneva Disponible en: http://www.who.int/tobacco/ mpower/mpower.flyerA4.web.SP.pdf?ua=1 
Organización Mundial de la Salud. (2015). La OMS pide más impuestos sobre el tabaco. OMS/OPS. Washington D.C Disponible http://www.elmundo.es/2015/07/07/559b89 bf46163f22728b4570.html

Suárez, L.N. Galceran, S. V. (2018). Normas jurídicas de prevención, control del tabaquismo y Convenio Marco para el Control del Tabaco en Cuba. Rev. Horizonte sanitario / vol. 17, no. 3, septiembre - diciembre 2018Disponible en: http://revistas.ujat.mx/index.php/horizonte

5. Baskerville, B. Et. al. (2015). Impact of Canadian tobacco packaging policy on quit line reach and reach equity. Prev Med; 81:243-50. Disponible en: http: //dx.doi.org/10.1016/j. ypmed.2015.09.010.

León DD, Ferreira PS, Pillon SC. (2010). Conocimientos y prácticas sobre el consumo de tabaco en estudiantes de pregrado de farmacia, Lima, Perú. Rev Latino-Am Enfermagem. Disponible en: http://www.scielo.br/scielo.php?script=sci_arttext\&pid=S0104$11692010000700014 \& \operatorname{lng}=\mathrm{es} \& n r m=\mathrm{iso} \& \operatorname{tng}=\mathrm{es}$

Llopiz, K. (2018). Educación antitabáquica en escolares primarios desde la labor extensionista universitaria. Rev. EDUMECENTRO, Vol. 10 (3). Disponible en: http://www.revedumecentro.sld.cu

Llopiz, K. (2018). La labor extensionista como vínculo entre las universidades de la provincia de Villa Clara. Rev. EDUMECENTRO, Vol. 10 (3). Disponible en: http://www.revedumecentro.sld.cu

Pupo, A. N. Hechavarría, T.S. Lozano, L.A. (2016). Caracterización de las publicaciones sobre tabaquismo en revistas científicas cubanas (2005-2014). Rev Cubana de Salud Pública; Vol 42(3). Disponible en: http://scielo.sld.cu/scielo.php?script=sci_arttext\&pid=S086434662016000300005

Rodríguez Fernández MC, Cruz Rodríguez J. (2015). La necesaria relación intersectorial educación-salud en la lucha contra las drogodependencias. EDUMECENTRO; Vol. 7(4). Disponible en: http://scielo.sld.cu/scielo.php?script=sci_arttext\&pid=S207728742015000400017

Suárez, L.N. (2016). Consumo de cigarrillos y elasticidad precio- demanda. Cuba. 2016. Rev Horizonte Sanitario [Internet]. 2017, [citado 04 de septiembre de 2017]; 16(1):8-14. Disponible en: http://revistas.ujat.mx/index.php/horizonte

Suárez L.N. (2014). Mercado y consumo de cigarrillos en Cuba y la decisión entre tabaco o salud. Rev. Cubana Salud Pública [Internet]. 40(3). Disponible en: http://scielo.sld.cu/scielo.php?script=sci_arttext\&pid=S086434662014000300003 